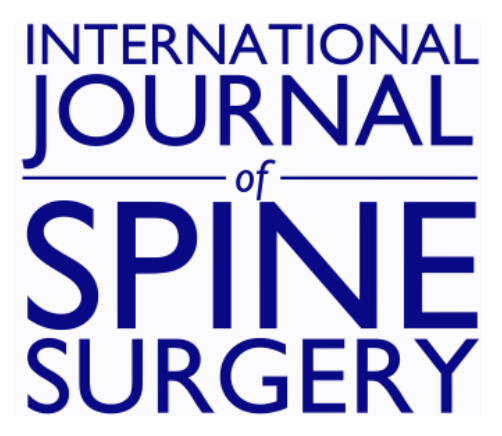

\title{
Lumbar Dorsal Root Ganglia Location: An Anatomic and MRI Assessment
}

Michael P Silverstein, Lynn J Romrell, Edward C Benzel, Nicolas Thompson, Sandra Griffith and Isador H Lieberman

Int J Spine Surg 2015, 9 ()

doi: https://doi.org/10.14444/2003

http://ijssurgery.com/content/9/3

This information is current as of April 26, 2023.

Email Alerts Receive free email-alerts when new articles cite this article. Sign up at: http://ijssurgery.com/alerts 


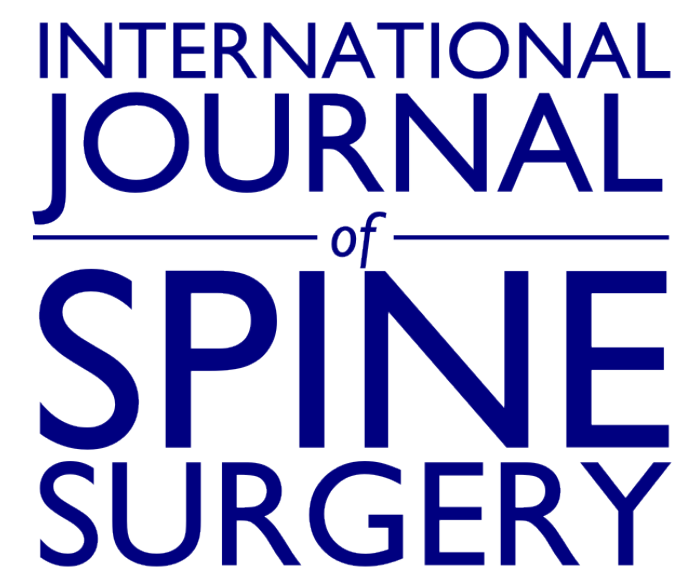

This article generously published free of charge by the International Society for the Advancement of Spine Surgery.

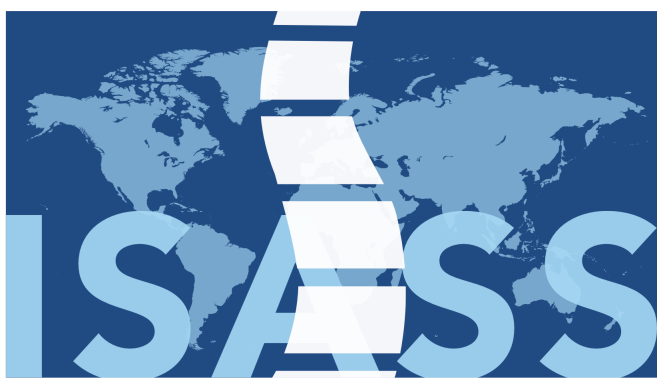

INTERNATIONAL SOCIETY for the ADVANCEMENT of SPINE SURGERY 


\section{Lumbar Dorsal Root Ganglia Location: An Anatomic and MRI Assessment.}

Michael P Silverstein, MD ${ }^{1,2}$, Lynn J Romrell, PhD ${ }^{3,}$ Edward C Benzel, MD, ${ }^{2,4}$ Nicolas Thompson, $P h D,{ }^{5}$ Sandra Griffith, PhD, ${ }^{5}$ Isador H Lieberman, $M D, M B A^{6}$

${ }^{1}$ Department of Orthopaedic Surgery, Cleveland Clinic Foundation, Cleveland, OH. ${ }^{2}$ Center for Spine Health, Cleveland Clinic Foundation, Cleveland OH. ${ }^{3}$ Department of Anatomy, College of Medicine, Florida State University, Tallahassee, FL. ${ }^{4}$ Department of Neurological Surgery, Cleveland Clinic Foundation, Cleveland, $\mathrm{OH} .{ }^{5}$ Department of Quantitative Health Sciences, Cleveland Clinic Foundation, Cleveland, OH. ${ }^{6}$ Scoliosis and Spine Tumor Center, Texas Back Institute, Plano, TX.

\section{Abstract}

\section{Background}

The dorsal root ganglion (DRG) is a key structure in the mechanism of symptomatic radicular pain, weakness and change in sensation. DRG localization can assist in the decision making process of which areas require decompression, and type of procedure that should be performed to treat radicular symptoms. In this study we determine dimensions of lumbar foramina, DRG and its relationship to the neuroforamina through anatomic and magnetic resonance imaging (MRI) evaluation Agreement between MRI and anatomic assessment of DRG location will be determined.

\section{Methods}

Sixteen embalmed cadavers, 10 females and 6 males, aged 68 to 106 years had an MRI of the thoracolumbar spine followed by dissection. Measurements made included foraminal height and width, DRG size and nerve root take off angle. The center of the DRG and its relationship to the foramina were measured and the probability of agreement between anatomic and MRI assessment were made.

\section{Results}

The greatest width of the DRG was $6.5 \mathrm{~mm}$ bilaterally at L5 (range $3.2-6.5 \mathrm{~mm}$ ). The nerve root take off angle was largest at L5 on the left (range 50.5o-58.8o) and L4 on the right (range 50.5o-57.2o). The center of the DRG was found bilaterally in the medial zone of the foramen of L1-4 and lateral zone at L5. Foramina size increased from L1 to L5 in the ventral to dorsal and cephalad to caudal direction. Pedicle width increased from L1 to L5. The estimated overall probability of agreement between anatomic and MRI DRG location was $86.3 \%$ ( $95 \%$ confidence interval $=77.5 \%-92.0 \%)$. 


\section{Conclusion}

The percentage of agreement between MRI and anatomic evaluation of lumbar DRG location significantly exceeded our pre-defined threshold of $70 \%(p=0.0013)$.

\section{Clinical Relevance}

Our results aid in surgical decision-making as true anatomic position can be directly correlated to what's seen on MRI.

keywords: lumbar spine, dorsal root ganglia, MRI, lumbar foramina, cadaver

Volume 9 Article 3 doi: 10.14444/2003

\section{Background}

The dorsal root ganglion (DRG) is a key structure in the mechanism of symptomatic radicular pain, weakness and change in sensation. ${ }^{1}$ The neuroforaminal and extraforaminal regions are two areas of increased risk for nerve and DRG entrapment by disc herniations or degenerative osteophytes as the nerve root exits the spinal canal. DRG localization can assist in the decision making process of which areas require decompression, and type of procedure that should be performed to treat radicular symptoms. With the advancement of minimally invasive procedures and their increased utilization in thoracolumbar and lumbar surgery precise knowledge of nerve anatomy and its spatial arrangements are particularly relevant. ${ }^{2,3}$ Such knowledge can be used to decrease the incidence of nerve root damage.

In an effort to further enhance our anatomic knowledge of DRG location and its anatomic relationships we carried out this study to determine the foraminal and DRG measurements and the location of the DRG in relationship to the neuroforamina. This was performed with anatomic and magnetic resonance imaging (MRI). We also assessed the agreement between MRI and anatomic assessment of DRG.

\section{Materials and Methods}

Sixteen embalmed cadavers, 10 females and 6 males, aged 68 to 106 years old (mean age 85.7) were included in the study. Prior to dissection, the thoracolumbar spine specimens were evaluated with MRI. The cadavers were then dissected and anatomical measurements were made. These measurements include foraminal height and width, DRG size and nerve root take off angle. The center of the DRG was determined based on the widest portion of the nerve root sleeve. The center of the DRG and its relationship to the foramina were measured. This was determined by the location of the center of the DRG in relation to intraspinal, neuroforaminal or extraforaminal region. ${ }^{4}$ The neuroformina was bisected in a medial to lateral fashion to create medial and lateral regions (Figure 1). 


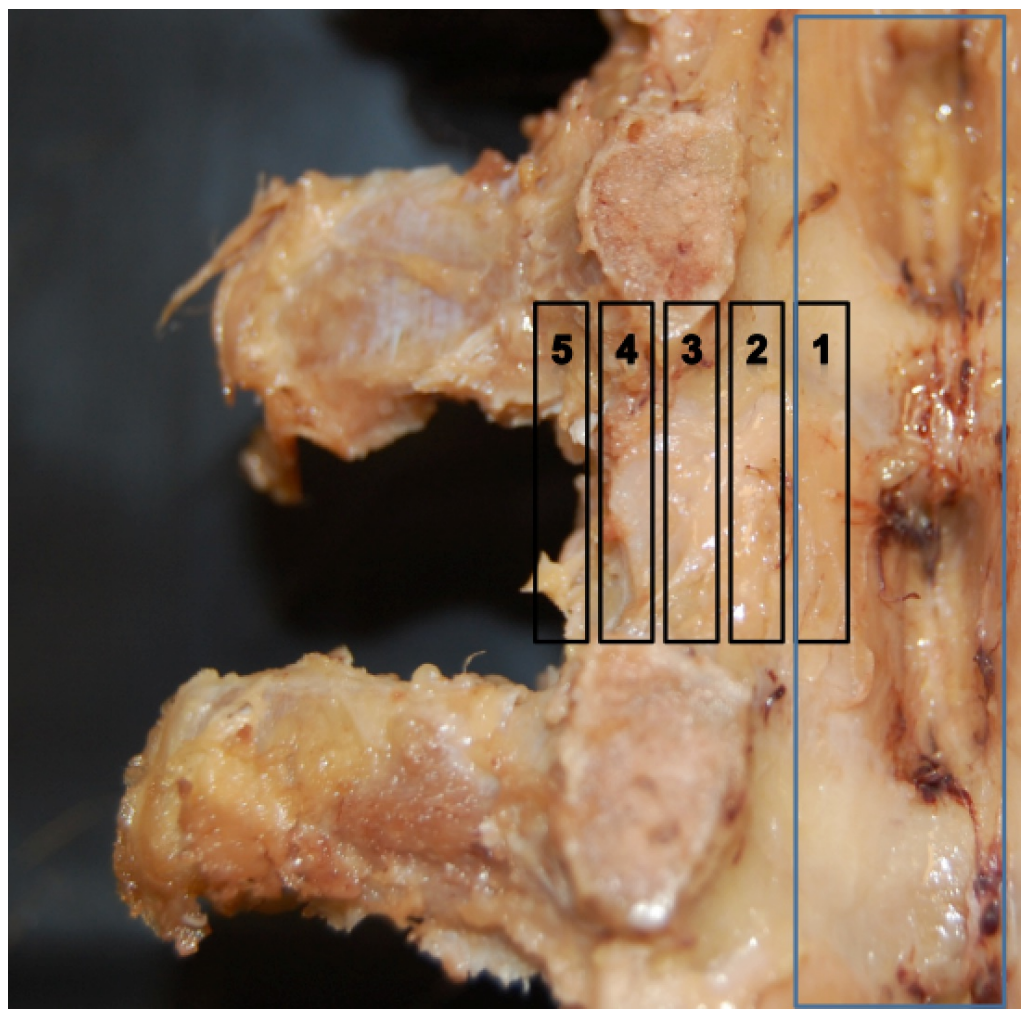

Fig 1. DRG region. Zone 1 and 2: intraspinal; Zone 3: medial neuroforamina; Zone 4: lateral neuroforamina; Zone 5: extraforaminal space.

\section{Statistical Methods}

To estimate the probability of agreement between anatomical examination and MRI, we created a mixed-effects logistic regression model, with the intercept removed. The response variable, which is agreement, was defined by whether the exact same positions were identified by both diagnostic measures for a given side (right or left) within one of the five levels of the specimen's lumbar spine. We included "Spinal Level" as a fixed effect (treated as categorical) and included a random effect for each specimen and a random effect for "side" (right verse left) within each specimen. Due to a large amount of missing data for L1, we excluded L1 values from analysis. Thus, the results for this study are only valid for L2-L5. We estimated the percent agreement within each spinal level and computed $95 \%$ confidence intervals. To obtain an overall level of agreement, we took a weighted average of the coefficients for each category of the variable Level and transformed from the logit scale to probability. A 95\% confidence interval for this predicted probability was then computed. We hypothesized the overall level of agreement would be significantly greater than $70 \%$. 


\section{Results}

There were 16 cadavers included in the analysis. For the MRI portion of the study, 10 cadavers were missing information for L1. One specimen was missing information for both right and left L2 and one specimen was missing information for the left side of L2. Missing information resulted from injury to the specimen. For all other spinal levels, complete information was available for each specimen.

The greatest width of the DRG was $6.5 \mathrm{~mm}$ both on the left and right of L5 (range 3.2-6.5mm)(Figure 1). The nerve root take off angle was largest at L5 on the left (range $50.5^{\circ}-58.8^{\circ}$ ) and $\mathrm{L} 4$ on the right (range $50.5^{\circ}-57.2^{\circ}$ ). The center of the DRG was found bilaterally in the medial zone of the foramen of L1-4 and the lateral zone at L5. (Figure 2) The foramina increased in size from L1 to L5 in both the ventral to dorsal and cephalad to caudal (pedicle to pedicle) direction. Pedicle width (medial to lateral) increased in size from L1 to L5 (Table 1).

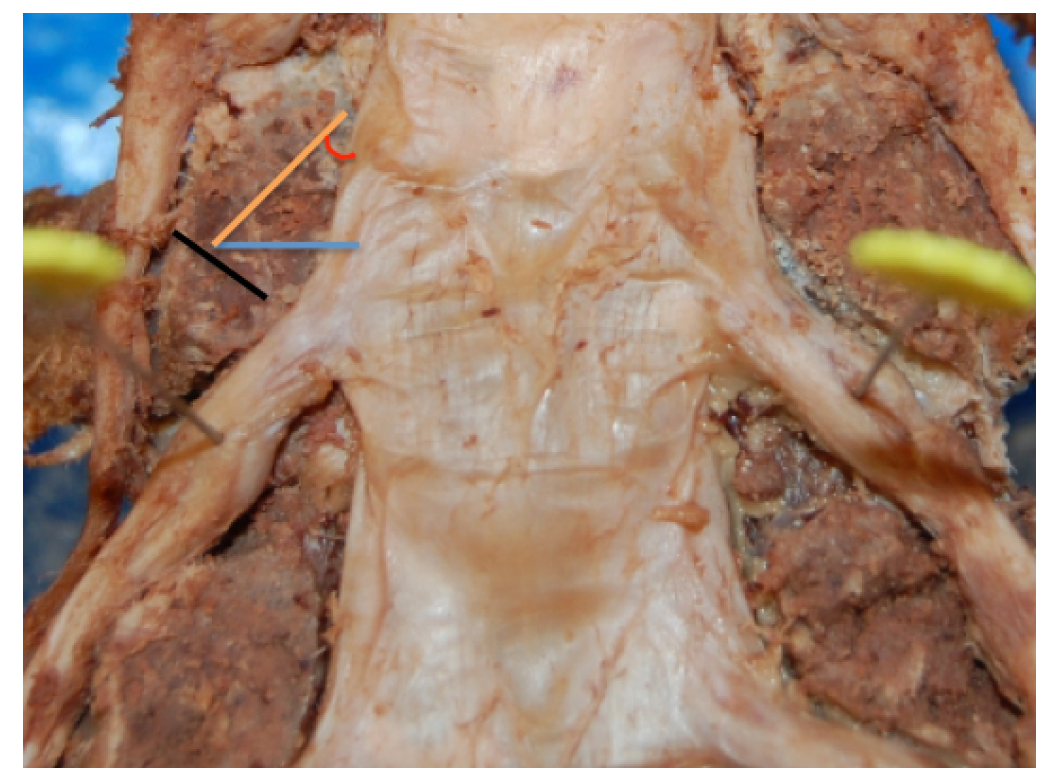

DRG Size (mm). Width is the width of DRG at the pin (maximal width of DRG). The nerve root angle is determined from $\sin (\mathrm{O})$ calculated by the right/diagonal lines relative to the width.

Table 1. Lumbar Spine Demographic.

\begin{tabular}{|c|c|c|c|c|c|c|c|c|c|c|}
\hline \multirow[t]{2}{*}{ DRG Size $(\mathrm{mm})$} & \multicolumn{5}{|c|}{ Left } & \multicolumn{5}{|c|}{ Right } \\
\hline & L1 & L2 & L3 & L4 & L5 & $\mathrm{L} 1$ & L2 & L3 & L4 & L5 \\
\hline Width: mean & 3.2 & 4.3 & 4.9 & 6.1 & 6.5 & 3.2 & 4.3 & 4.9 & 6.1 & 6.5 \\
\hline Width: sem & 0.7 & 0.4 & 0.3 & 0.4 & 0.5 & 0.7 & 0.4 & 0.3 & 0.4 & 0.5 \\
\hline \multirow[t]{2}{*}{ DRG Region } & \multicolumn{5}{|c|}{ Left } & \multicolumn{5}{|c|}{ Right } \\
\hline & $\mathrm{L} 1$ & L2 & L3 & L4 & L5 & $\mathrm{L} 1$ & L2 & L3 & L4 & L5 \\
\hline Zone: mean & 3.0 & 3.0 & 3.5 & 3.9 & 4.3 & 3.0 & 3.1 & 3.5 & 3.8 & 4.2 \\
\hline Zone: sem & 0.3 & 0.1 & 0.1 & 0.1 & 0.1 & 0.3 & 0.2 & 0.1 & 0.1 & 0.1 \\
\hline \multirow[t]{2}{*}{ Intervertebral Foramina Size (mm) } & \multicolumn{5}{|c|}{ Left } & \multicolumn{5}{|c|}{ Right } \\
\hline & $\mathrm{L} 1$ & L2 & L3 & L4 & L5 & $\mathrm{L} 1$ & $\mathrm{~L} 2$ & L3 & L4 & L5 \\
\hline AP: mean & 6.6 & 7.4 & 7.6 & 8.4 & 8.7 & 6.8 & 7.2 & 7.4 & 8.5 & 8.6 \\
\hline
\end{tabular}




\begin{tabular}{|l|l|l|l|l|l|l|l|l|l|l|}
\hline AP: sem & 0.2 & 0.2 & 0.3 & 0.4 & 0.4 & 0.4 & 0.2 & 0.3 & 0.2 & 0.4 \\
\hline PP: mean & 7.2 & 8.1 & 9.4 & 10.1 & 10.1 & 7.3 & 8.5 & 9.4 & 10.4 & 10.3 \\
\hline PP: sem & 1.8 & 0.7 & 0.8 & 0.8 & 0.7 & 1.8 & 0.7 & 0.8 & 0.8 & 0.7 \\
\hline Pedicle width (medial to lateral) (mm) & Left & & & & Right & & \\
\hline & L1 & L2 & L3 & L4 & L5 & L1 & L2 & L3 & L4 & L5 \\
\hline Width: mean & 7.0 & 7.0 & 7.8 & 8.7 & 9.7 & 6.7 & 7.1 & 7.9 & 8.9 & 9.7 \\
\hline Width: sem & 0.3 & 0.3 & 0.2 & 0.3 & 0.4 & 0.2 & 0.3 & 0.2 & 0.4 & 0.4 \\
\hline Nerve Root Angle & Left & & & & & Right & & \\
\hline & L1 & L2 & L3 & L4 & L5 & L1 & L2 & L3 & L4 & L5 \\
\hline Angle (rounded to nearest 10th) & 50.5 & 52.2 & 55.2 & 57.4 & 58.8 & 50.5 & 52.2 & 55.2 & 57.2 & 56.3 \\
\hline
\end{tabular}

Key: DRG Region zone 1 and 2: intraspinal; zone 3: medial neuroforamina; zone 4: lateral neuroforamina; zone 5: extraforaminal space. Intervertebral Foraminal Size. Anteriorposterior (AP): maximal distance from posterior body/disk to facet joint. Pedicle to pedicle (PP) distance: inferior border of upper to superior border of lower pedicle. SEM: standard error of the mean. Angle determined via $\sin (\mathrm{O})=\mathrm{right} /$ diagonal.

Table 2 displays raw percent agreement for each level, both as a raw percentage and adjusted values obtained from the logistic regression model. Recall that the L1 level was not included in the regression model. As Level increased from 2-5, the model-adjusted percent agreement increased from $77.4 \%$ (Level 2) to $94.4 \%$ (Level 5). After adjusting for Level and correlated responses within specimens and side within each specimen, the estimated overall probability of agreement was $86.3 \%$ (95\% confidence interval $=77.5 \%$ $-92.0 \%)$. This percentage of overall agreement significantly exceeded our pre-defined threshold of $70 \%(\mathrm{p}=0.0013)$.

Table 2.

\begin{tabular}{|l|l|l|l|}
\hline \multicolumn{2}{|l|}{ Raw Values } & \multicolumn{2}{l|}{ Model-Adjusted Values } \\
\hline Level & Proportion Agree & \% Agree & $\begin{array}{l}\text { \% Agree } \\
\text { (95\% Confidence Interval) }\end{array}$ \\
\hline 1 & $8 / 12$ & 66.7 & ---- \\
\hline 2 & $22 / 29$ & 75.9 & $77.4(58.0,89.5)$ \\
\hline 3 & $25 / 32$ & 78.1 & $79.4(61.2,90.4)$ \\
\hline 4 & $28 / 32$ & 87.5 & $88.6(71.9,95.9)$ \\
\hline 5 & $30 / 32$ & 93.8 & $94.4(79.0,98.7)$ \\
\hline
\end{tabular}

\section{Discussion}

Lumbar disc herniation and degenerative osteophytes are a major cause of acute and chronic radicular symptoms, due to dorsal root ganglia compression. ${ }^{5-7}$ Patient history, physical examination findings and advanced imaging (ie MRIs) are used in the diagnosis of spinal disorders. They can show DRG entrapment and the regional position, which can be helpful when choosing the appropriate surgical intervention. The location of the DRG can be divided into intraspinal, neuroforaminal and extraforaminal regions. We were able to quantify anatomic measurements of the DRG, foramina and their relationship. The location of the DRG determined from anatomical and MRI evaluation proved to share an overall probability of agreement of $86.3 \%$. We found that the DRG is positioned more lateral from L1 through L5. 
DRG location and symmetry may be altered by surrounding structures. ${ }^{8}$ Hamanishi found 30 pairs among 221 pairs of lumbar DRG to be asymmetric on MRI. For example, asymmetry can be seen on MRI as a result of a herniated disc stretching the nerve root posteriorly changing the DRG location. Tissue hypertrophy, arthropathy, scoliosis and congenital conditions can be an etiologic cause as well. We found 2 of 68 pairs and 12 of 68 pairs of lumbar DRG to be asymmetric from anatomic and MRI evaluation, respectively.

Our study found a high percentage of L5 DRGs to be located in the neuroformainal space. Kikuchi et $\mathrm{al}^{9}$ evaluated DRG location of the lower lumbar spine in a comparison study of 22 cadavers undergoing anatomic and radiographic assessment (with nerve root enhancement). He determined the anatomic location of L5 DRG to be $19.2 \%$ intraspinal, $72.8 \%$, intraformainal, and $8 \%$ extraforminal compared to $38.9 \%$ intraspinal, $52.7 \%$ intraforaminal and $8.4 \%$ extraforminal based on radiograph assessment $(p<0.5)$. The slight variation in findings from Kikuchi is twofold. Kikuchi et al. measures the DRG location based on the proximal end of the DRG. We determined the location based on the widest portion of DRG within the particular zone. Also, there is a slight variation in the fashion of zone designation.

A higher incidence of foraminal stenosis is found in the lower lumbar segments. Jenus and An determined the most common entrapped roots are L5 (75\%), L4 (15), L3 (5\%) and L2 (4\%). ${ }^{10,11}$ Our data shows the L4 and L5 levels to contain the DRG in the neuroformina. This is important when planning a surgical approach. For example, when the patient's symptoms are a result of pure foraminal stenosis an extraforaminal decompression using a paramedian Wiltse type approach with foraminotomy can be most appropriate. $^{12}$

While the Wiltse paramedian approach is a common procedure for performing foraminal decompression, endoscopic transforaminal decompression has gained much popularity. ${ }^{13-15}$ Transforaminal approach provides access for formainal decompression with less destabilization compared to a midline and Wiltse approach. ${ }^{16}$ Endoscopic transforaminal decompression, while used during primary decompression, also has been utilized for revision lumbar surgery. ${ }^{17}$ Under endoscopic foramen visualization, stenosis can be seen as an absence of fat, vascular pulsation with scarred, fibrotic nerve roots in the axilla between the exiting and traversing nerves. The is area is known as the hidden zone of McNab. ${ }^{17,18}$ Our study provides valuable anatomic knowledge as endoscopic transformainal decompression utilizes a small window of visualization. Foraminal zones as described in this study can be accessed with a transformainal approach from lateral to medial. Careful evaluation of pre-operative MRI findings will provide spatial relationships between the DRG and its surrounding structures. In addition to MRI, CT imaging with sagittal and coronal reconstruction can further aid in spatial assessment.

Pedicle width is noted to increase in size from L1 to L5 in our study. Kadioglu et al ${ }^{19}$ found the pedicle width to increase from L1 to L5 as well. They reported pedicle width to range from 4 to $14 \mathrm{~mm}$. As pedicle width increases from L1 to L5, the neuroforamina width will increase as well. Thus, it is more likely that the DRG will be found within the neuroforamina in the lower lumbar spine. Implication of this would include the need for a smaller verses larger foraminotomy to create space following DRG impingement. 
Steroid injections are commonly performed for treatment of radiculopathy. ${ }^{20-22}$ The location of administration may vary, however. DRG compression can be the etiology for symptoms. Hence, MRI verification of the DRG location can help with procedural planning. Also, MRI can be used for guidance imaging as well. ${ }^{23} \mathrm{With}$ our results of a greater than $70 \%$ agreement the DRG location determined from MRI is similar to anatomic evaluation increases the likelihood medication infiltration will be at the appropriate location around the DRG.

Lumbar DRG motion is dynamic with slight positional change with activity. Smith et $\mathrm{al}^{24}$ documented up to $5 \mathrm{~mm}$ of linear displacement in unembalmed cadavers during straight leg raise (SLR) maneuver following laminectomy with facetectomy. They found movement to increase when hip flexion increased from $30^{\circ}$ of flexion. During intraoperative assessment following lumbar discectomy, Kobayashi et $\mathrm{al}^{25}$ found up to $4 \mathrm{~mm}$ of root displacement. Grimes et $\mathrm{al}^{26}$ described 4 distinct ligamentous bands connecting the root sleeve to the surrounding foraminal structures. These attachments are suggested to restrict distal displacement of the nerve roots. Thus, dissections performed by Smith and Kobayashi may in fact exaggerate movement. Gilbert et $\mathrm{al}^{27}$ found the L4, L5 and S1 to move on average less than $1 \mathrm{~mm}$ during SLR when hip flexion was greater than $60^{\circ}$ with intact foraminal ligaments in unembalmed cadavers. Thus, this little motion which can also relate to standing and sitting would likely result in the DRG remaining within the neuroforamen.

In this study we were able to describe the spatial arrangement of the dorsal root ganglia within the lumbar spine. In addition, we were able to quantify the foraminal dimensions and nerve root take off angles which both contribute to our understanding of the anatomical relationships of the lumbar spine.

While we were able to determine MRI and anatomic agreement, the study did face some limitations. The sample size was small and some levels were unable to be included in analysis secondary to poor anatomic and MRI visualization. Further evaluation could increase the power of this study. With changes in fat composition and lack of CSF in cadavers, there may be a slight change in the DRG location.

\section{Conclusion}

The percentage of overall agreement between MRI and anatomic evaluation of lumbar DRG location significantly exceeded our pre-defined threshold of $70 \%(p=0.0013)$. Our results can aid in surgical decision-making as true anatomic position can be directly correlated to what is seen on MRI.

\section{References}

1. Kobayashi S, Yoshizawa H, Yamada S. Pathology of lumbar nerve root compression. Part 2: morphological and immunohistochemical changes of dorsal root ganglion. J Orthop Res. 2004;22(1):180-8.

2. Dahdaleh NS, Smith ZA, Hitchon PW. Percutaneous pedicle screw fixation for thoracolumbar fractures. Neurosurg Clin N Am. 2014;25(2):337-346.

3. Wong AP, Smith ZA, Stadler JA, et al. Minimally invasive transforaminal lumbar interbody fusion (MI-TLIF): surgical technique, long-term 4-year prospective 
outcomes, and complications compared with an open TLIF cohort. Neurosurg Clin N Am. 2014;25(2):279-304.

4. Shen J, Wang HY, Chen JY, el al. Morphologic analysis of normal human lumbar dorsal root ganglion by 3D MR imaging. AJNR Am J Neuroradiol. 2006;27(10):2098-103.

5. Weber H. Lumbar disc herniation. A controlled, prospective study with ten years of observation. Spine 1983;8(2):131-40.

6. Carragee EJ, Kim DH. A prospective analysis of magnetic resonance imaging findings in patients with sciatica and lumbar disc herniation. Correlation of outcomes with disc fragment and canal morphology. Spine 1997;22(14):1650-60.

7. Jacobs WC, Van Tulder M, Arts M, et al. Surgery versus conservative management of sciatica due to a lumbar herniated disc: a systematic review. Eur Spine J. 2011;20(4):513-22.

8. Hamanishi C, Tanaka S. Dorsal root ganglia in the lumbosacral region observed from the axial views of MRI. Spine 1993;18(13):1753-6.

9. Kikuchi S, Sato K, Konno S, et al. Anatomic and radiographic study of dorsal root ganglia. Spine 1994;19(1):6-11.

10. Jenis LG, An HS. Spine update. Lumbar foraminal stenosis. Spine 2000;25(3):389-94.

11. Van Roy P, Barbaix E, Clarijs JP, et al. Anatomical background of low back pain: variability and degeneration of the lumbar spinal canal and intervertebral disc. Schmerz. 2001;15(6):418-24.

12. Vialle R, Wicart $\mathrm{P}$, Drain $\mathrm{O}$, et al. The Wiltse paraspinal approach to the lumbar spine revisited: an anatomic study. Clin Orthop Relat Res. 2006;445:175-80.

13. Jasper GP, Francisco GM, Telfeian AE. Clinical success of transforaminal endoscopic discectomy with foraminotomy: a retrospective evaluation. Clin Neurol Neurosurg. 2013;115(10):1961-5.

14. Jang JS, An SH, Lee SH. Transforaminal percutaneous endoscopic discectomy in the treatment of foraminal and extraforaminal lumbar disc herniations. J Spinal Disord Tech. 2006;19(5):338-43.

15. Hoogland T, Van den brekel-dijkstra K, Schubert M, et al. Endoscopic transforaminal discectomy for recurrent lumbar disc herniation: a prospective, cohort evaluation of 262 consecutive cases. Spine 2008;33(9):973-8.

16. Osman SG, Nibu K, Panjabi MM, et al. Transforaminal and posterior decompression of the lumbar spine. A comparative study of stability and intervertebral foramen area. Spine 1997;22(15):1690-5.

17. Yeung AT, Gore S. Endoscopic foraminal decompression for failed back surgery syndrome under local anesthesia. Int J. Spine Surg. 2014;8(22): 1-20.

18. Yeung AT, Gore S. In-vivo endoscopic visualization of pathoanatomy in symptomatic degenerative conditions of the lumbar spine II: intradiscal, foraminal, and central canal decompression. Surg Technol Int. 2011;XXI:299-319.

19. Kadioglu HH, Takci E, Levent A, et al. Measurements of the lumbar pedicles in the Eastern Anatolian population. Surg Radiol Anat. 2003;25(2):120-6.

20. Slipman CW, Chow DW. Therapeutic spinal corticosteroid injections for the management of radiculopathies. Phys Med Rehabil Clin N Am. 2002;13(3):697-711.

21. Staal JB, De bie RA, De vet HC, et al. Injection therapy for subacute and chronic low back pain: an updated Cochrane review. Spine 2009;34(1):49-59. 
22. Berger O, Dousset V, Delmer O, et al. Evaluation of the efficacy of foraminal infusions of corticosteroids guided by computed tomography in the treatment of radicular pain by foraminal injection. J Radiol. 1999;80(9):917-25.

23. Fritz J, Sequeiros RB, Carrino JA. Magnetic resonance imaging-guided spine injections. Top Magn Reson Imaging 2011;22(4):143-51.

24. Smith SA, Massie JB, Chesnut R, et al. Straight leg raising. Anatomical effects on the spinal nerve root without and with fusion. Spine. 1993;18(8):992-9.

25. Kobayashi S, Shizu N, Suzuki Y, et al. Changes in nerve root motion and intraradicular blood flow during an intraoperative straight-leg-raising test. Spine. 2003;28(13):1427-34.

26. Grimes PF, Massie JB, Garfin SR. Anatomic and biomechanical analysis of the lower lumbar foraminal ligaments. Spine. 2000;25(16):2009-14.

27. Gilbert KK, Brismée JM, Collins DL, et al. 2006 Young Investigator Award Winner: lumbosacral nerve root displacement and strain: part 1. A novel measurement technique during straight leg raise in unembalmed cadavers. Spine. 2007;32(14):1513-20.

\section{Disclosures}

Isadore Lieberman, MD, MBA's full list of disclosures follows: royalties, IP rights, consulting, ownership interest, management, founder: Merlot OrthopediX Inc; royalties, IP rights, consulting, ownership interest: Axiomed Spine Corporation; royalties, IP rights, consulting, ownership interest, Mazor Surgical Technologies; Royalties, Stryker Spine; Consulting, Bionik Laboratories; Consulting, Globus Medical Inc, Speaker, J\& J DePuy Synthes. The other authors report no relevant disclosures.

\section{Corresponding Author}

Michael P Silverstein, MD, 12911 Cedar Road, Cleveland Heights, OH

44118.msilve28@gmail.com

Published 12 February 2015.

Copyright (C) 2015 ISASS - International Society for the Advancement of Spine Surgery. To see more or order reprints or permissions, see http://ijssurgery.com. 